\title{
Inventario florístico en el cerro Tetzcotzinco, Texcoco, Estado de México ${ }^{1}$
}

\author{
Ma. Teresa P. Pulido y Stephen D. $\mathrm{KOCH}^{2}$
}

\begin{abstract}
RESUMEN. El cerro Tetzcotzinco es un sitio de importancia arqueológica y atractivo turístico, ubicado cerca de Texcoco, Estado de México. Es de origen volcánico, con laderas empinadas y rocosas, y cuya altitud va de 2270 a $2600 \mathrm{msnm}$. Se reconocen cuatro tipos de agrupaciones vegetales en el cerro: bosque de encinos, pastizal, matorral xerófilo y vegetación acuática y subacuática. El inventario realizado revela la presencia de 375 especies, 234 géneros y 70 familias de plantas vasculares, una diversidad que sorprende en un área de 50 ha. La mayoría de estas especies es conocida del Valle de México, pero hubo hallazgos notables. Entre éstos destacan dos: Pavonia pulidoae, una especie nueva, y Thevetia peruviana, una especie muy poco conocida en el Valle de México.
\end{abstract}

AbSTRACT. Cerro Tetzcotzinco is an important archeological site and tourist attraction located near Texcoco, State of Mexico. It is a hill of volcanic origin, with steep rocky slopes and an elevation of $2270-2600 \mathrm{~m}$. Four plant associations are recognized on the hill, oak woods, grassland, xeromorphic scrub, and aquatic and semiaquatic vegetation. The inventory revealed the presence of 375 species, 234 genera, and 70 families of vascular plants, a surprising diversity for an area of 50 ha. The majority of these species are known from the Valley of Mexico, but there were notable discoveries. Outstanding among these are Pavonia pulidoae, a new species, and Thevetia peruviana, a species very little known in the Valley of Mexico.

\section{INTRODUCCIÓN}

El cerro Tetzcotzinco es uno de los atractivos turísticos de la región de Texcoco, Estado de México. Es un lugar muy visitado tanto para conocer sus restos arqueológicos como para disfrutar de su belleza natural. El área es muy reducida, pero contiene una alta diversidad botánica y su vegetación está en buenas condiciones, comparada con la de sus alrededores.

Como en muchas partes del Valle de México, la flora de este cerro está en peligro

\footnotetext{
${ }^{1}$ Parte de la tesis de licenciatura del primer autor, Guía ilustrada para la flora del cerro Tetzcotzinco, Estado de México, Facultad de Ciencias, Universidad Nacional Autónoma de México, 1982.

${ }^{2}$ Centro de Botánica, Colegio de Postgraduados, 56230 Chapingo, Edo. de México, México.
}

Pulido MTP, Koch SD. 1988. Inventario florístico en el cerro Tetzcotzinco, Texcoco, Estado de México. Boletín de la Sociedad Botánica de México 48: 81-94. 
de ser fuertemente alterada en corto tiempo debido a las actividades humanas que cada día deterioran la ecología del lugar. Además, ha sido poco explorado botánicamente, y la flora contiene un alto porcentaje de plantas útiles, principalmente medicinales. Así, el cerro reúne las tres características que la convierten en un área prioritaria para, inventariar su flora. Tales características, destacadas por Rzedowski en su ponencia de 1979 en el Coloquio sobre Estudios Florísticos y su Futuro en México, son: a) el lugar debe ser poco explorado; b) la flora del área debe estar en peligro de desaparecer por las actividades humanas, y c) la flora incluye un número importante de plantas útiles.

El objetivo principal de este estudio es inventariar las plantas vasculares del cerro Tetzcotzinco con los fines de contribuir al conocimiento de la flora del Valle de México, y de aportar datos de base para apoyar un intento de conservar el área. Un objetivo secundario es buscar evidencias florísticas de un jardín botánico prehispánico en este sitio, como se menciona en los relatos históricos.

\section{UBICACIÓN DE LA ZONA Y DATOS ABIÓTICOS}

El cerro Tetzcotzinco, localizado en los 98 $49^{\prime} 05^{\prime \prime}$ longitud Oeste y 19029'46" latitud Norte, es la parte más occidental de la Sierra del Tláloc. Constituye, además, el extremo sur de una pequeña cordillera semicircular que rodea los pueblos de La Purificación, San Miguel Tlaixpan y San Nicolás Tlamincas (fig. 1). Su gradiente altitudinal es de 2270 a $2600 \mathrm{msnm}$, y su área es de casi $50 \mathrm{ha}$. El acceso es por una carretera hacia el este de la ciudad de Texcoco en el Estado de México, a una distancia de siete kilómetros.

Ortiz y Cuanalo (1977) consideran esa pequeña cordillera como el complejo terrestre Tlaixpan, cuyas características fisiográficas son las siguientes: es una zona con laderas escarpadas, con pendientes de 40 a 50 por ciento. El material geológico es de rocas ígneas -andesitas, dasitas, anfibotes y basaltos. Su aspecto general es de conos y derrames ígneos erosionados geológicamente. El suministro hídrico se da por corrientes torrenciales de temporal, y algunos canales pequeños de manantiales y pozos profundos. Los suelos que se presentan son de profundidad variable, negros, de textura fina, pero con afloración de rocas en más de $40 \%$ de su superficie.

El clima de la zona se deduce de los datos de la estación metereológica en San Miguel Tlaixpan, base sobre la cual García (1978) lo describe como del tipo C(wo) (w) b (i'), es decir, templado y subhúmedo, con lluvias principalmente en el verano, con la temperatura media del mes más frío de entre $-3^{\circ}$ y $18^{\circ} \mathrm{C}$, y con poca oscilación anual de temperaturas medias. El promedio de precipitación anual es de 658 milímetros.

\section{ANTECEDENTES BOtÁNiCOS SOBRE EL CERRO TETZCOTZINCO}

La fama de este cerro se inicia desde que el rey-poeta Nezahualcóyotl (1402-1472) lo eligió como su residencia preferida, por lo cual lo enriqueció y lo embelleció con una gran diversidad de plantas, animales, pinturas y esculturas (Mendizábal, 1925).

Aparentemente se desarrolló una especie de jardín botánico en este sitio. Esta conclusión y todas las interpretaciones que se han hecho de las ruinas del cerro, se han basado en dos escritos del siglo XVI. Uno de ellos es el de Fernando de Alva Ixtlixóchitl (1955), escrito en 1600-1616 que al referirse a Tetzcotzinco dice: 


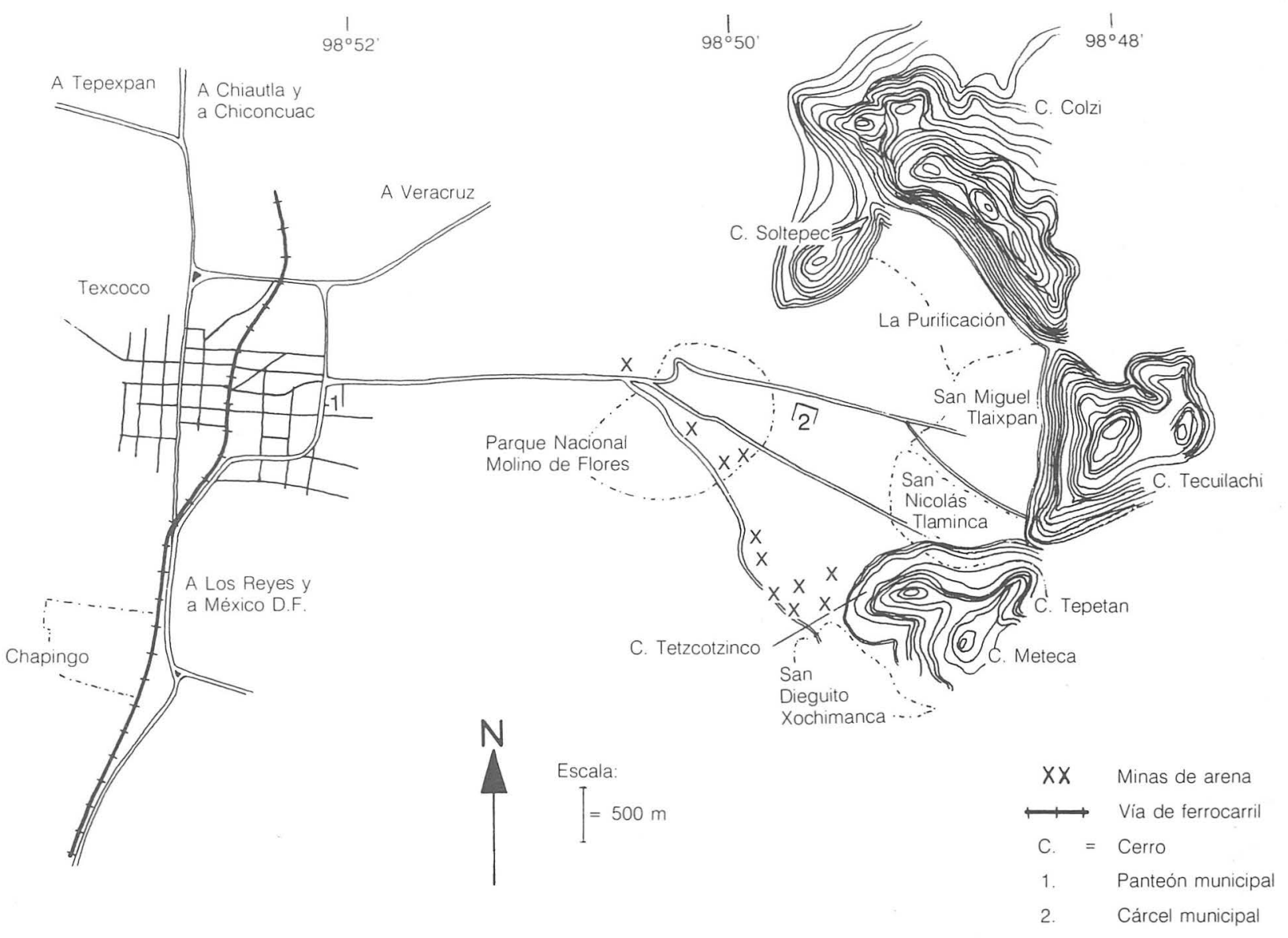

Fig. 1. Localización del cerro Tetzcotzinco. Tomado de Cetenal (1979 y 1979a) 
... el agua que se traía para las fuentes, pilas, baños y caños que se repartían para el riego de las flores y árboles de este bosque... de esta alberca salía un caño de agua que saltando sobre unas peñas salpicaba el agua, que iba a caer en un jardín de todas flores olorosas de tierra caliente... Todo lo demás de este bosque como dicho tengo, estaba plantado de diversidad de árboles y flores odoríferas; y en ellos diversidad de aves...

El otro es el de Juan Bautista Pomar (1975), escrito en 1582, menciona:

...en los cuales [jardines], tenían plantadas diversidad de flores y árboles de todas suertes, peregrinos y traídos de partes remotas...

Además, Maldonado Koerdell (1941) cita que había representaciones de plantas en las paredes que no podían mantenerse vivas aquí.

Paralelamente, se ha dilucidado que las instalaciones fungían como el centro de una zona de producción agrícola (Maldonado Koerdell, 1941). Según Corona (1978), se construyó un complejo hidráulico para aprovechar el agua traída al jardín para el riego constante de las terrazas que aún hoy rodean al cerro.

El único antecedente reciente sobre la flora del cerro es una lista mimeografiada, elaborada en 1976 por Engleman y Koch, como una guía de excursión. En ella, los autores hacen notar la gran riqueza florística del lugar y reportan las especies más comunes que atraen a la vista.

\section{VEGETACIÓN ACTUAL}

Toda la vegetación del cerro está perturbada en algún grado; de cualquier modo, podemos reconocer cuatro tipos de vegetación, tomando como base el sistema de Rzedowski y Rzedowski (1979) para el Valle de México (fig. 2). Éstos son:

Bosque de encinos. Esta área es un bosque bajo, entre 5 y 12 metros de alto, y caducifolio. La especie dominante es Quercus deserticola y hay algunos elementos de Alnus y Juniperus. Las epífitas son abundantes, representadas por cuatro especies de Tillandsia. En los estratos inferiores predominan géneros como Baccharis, Dablia, Desmodium, Eupatorium, Lamourouxia, Mublenbergia, Salvia, Senecio, Stevia, Thalictrum, y Valeriana. Este bosque cubre las laderas norte y oeste. En algunas de las partes más perturbadas hay matorral de Quercus frutex formando manchones.

Matorral xerófilo. La especie dominante es Eysenhardtia polystachya. Cubre casi la tercera parte del área total del cerro y se encuentra en las laderas sur y este. Abunda Phoradendron brachystachyum como epífita; en el estrato arbustivo predominan Montanoa tomentosa y Sedum praealtum; también hay Schinus molle y Opuntia spp., aunque estos últimos de ningún modo son abundantes.

Pastizal. No presenta una composición constante, pero las especies más abundantes son Hilaria cenchroides, Bouteloua simplex y Lycurus phleoides. Hay además elementos dispersos de matorral xerófilo como Opuntia streptacantha y Schinus molle. Se encuentra en la ladera suroeste de las faldas del cerro y en la ladera noreste cerca de la cima.

Vegetación acuática y subacuática. Existen elementos de este tipo de vegetación a lo largo de un angosto acueducto que funciona todo el año, según las necesidades de los agricultores. Entre los géneros más abundantes están Epilobium, Juncus, Lilaeopsis, Minulus y Ranunculus. 

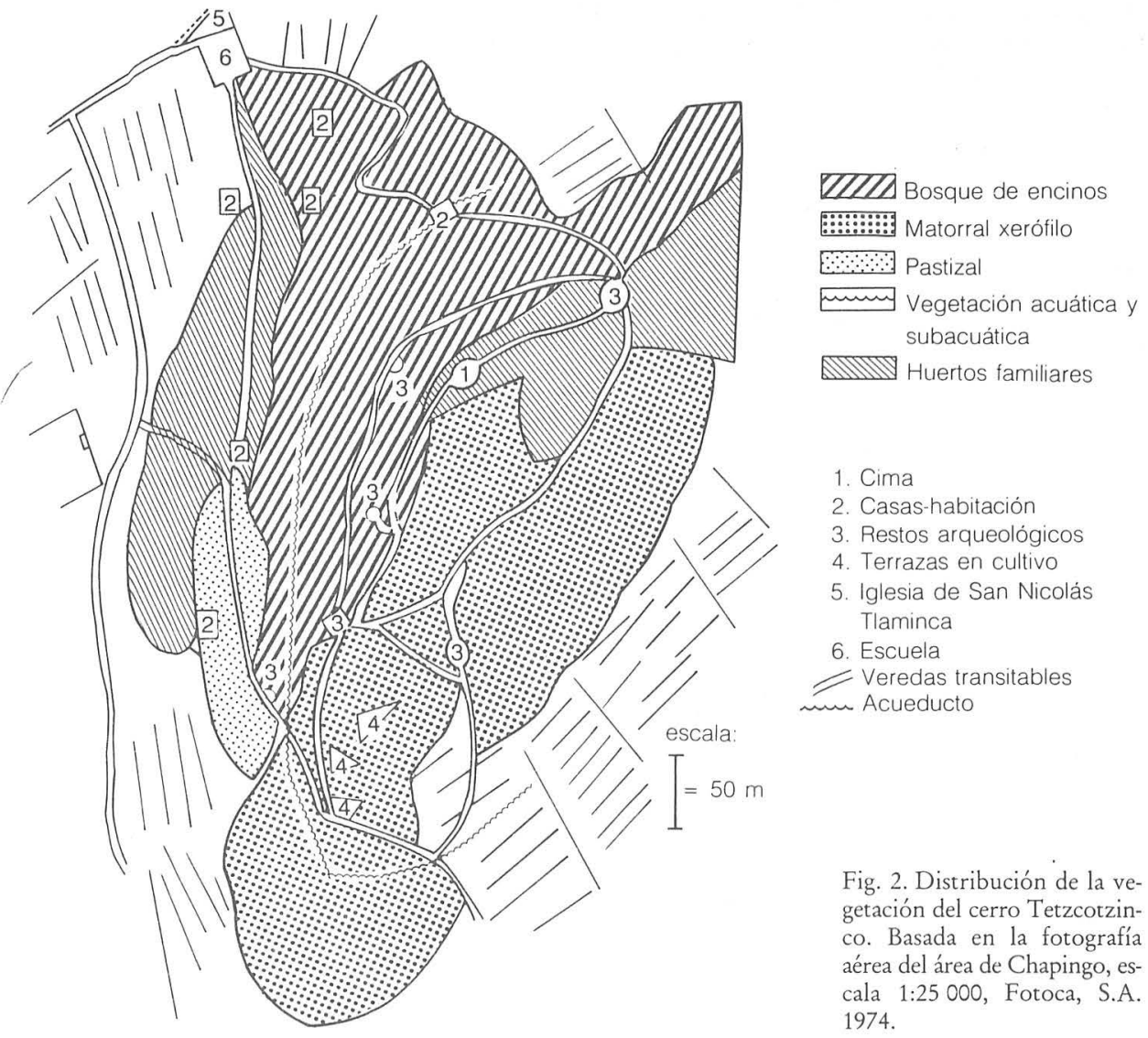

\author{
1. Cima \\ 2. Casas-habitación \\ 3. Restos arqueológicos \\ 4. Terrazas en cultivo \\ 5. Iglesia de San Nicolás \\ Tlaminca \\ 6. Escuela \\ Veredas transitables
Acueducto
}

Fig. 2. Distribución de la vegetación del cerro Tetzcotzinco. Basada en la fotografía aérea del área de Chapingo, escala 1:25000, Fotoca, S.A. 1974.

En los terrenos privados y cerca de las casas habitación existen muchas especies cultivadas. Tales especies no se tomaron en cuenta para este estudio.

\title{
Metodología
}

Para asegurar que la recolección fuera exhaustiva, se visitó el cerro dos o tres veces por semana durante 22 meses, y se hizo el esfuerzo de recorrer cada parte varias veces en cada estación del año. Se herborizaron ejemplares de todas las especies, hasta con cinco duplicados de cada colecta. El primer juego de esta colección está depositado en el Herbario-Hortorio del Colegio de Postgraduados, en Chapingo, Estado de México (CHAPA), el segundo en el Herbario de la Facultad de Ciencias, UNAM (FCME) y el resto fue distribuido por medio del programa de intercambio de CHAPA.

Las identificaciones fueron efectuadas por medio de la literatura' existente y de la 
comparación con ejemplares depositados en los herbarios locales, CHAPA, ENCB y MEXU, $\mathrm{y}$, en algunos casos, por consultas con especialistas.

\section{Resultados}

Se presenta el inventario completo de las plantas vasculares silvestres en el cerro Tetzcotzinco. A manera de resumen diremos que se obtuvieron los totales siguientes: 496 números en la recolección, 375 especies, 234 géneros y 70 familias.

En la tabla 1, se presenta un panorama general de la diversidad encontrada en cada uno de los tipos de vegetación.

\section{DisCUSIÓN}

Diversidad. La diversidad vegetal en este cerro es notoria a simple vista, si se compara con la de los cerros vecinos. Constituye uno de los principales atractivos para el visitante, lo que ha sido comprobado al inventariar las especies que la componen. Para hacer evidente aquí esta diversidad, se presenta la tabla 2, en la que se comparan los resultados de este estudio con algunos otros inventarios florísticos realizados para plantas vasculares en áreas más o menos similares.

Es notable que no obstante su área reducida, el cerro Tetzcotzinco tiene más especies, géneros y familias que la Sierra de Guadalupe o el campo experimental San Juan Tetla (Boyas, 1978). Solamente el Pedregal de San Ángel lo supera, pero el área de este último es muchas veces más grande.

La alta diversidad florística del cerro da origen a especulaciones acerca de cuáles condiciones especiales existen en el cerro que favorecen la diversidad florística. Se concluye que las más importantes son las siguientes:

1) El clima. Como ya hemos anotado, es templado y benigno como en la mayor parte del Valle, pero además el cerro tiene lugares siempre húmedos, cuando menos en sus laderas norte y oeste, debido al efecto de sombra durante la época seca.

2) La forma del cerro y su superficie rocosa. Las laderas del cerro son empinadas y abundan los riscos y afloramientos de roca que ofrecen una gran variedad de micrositios. También existen numerosos manantiales temporales, además del acueducto que funciona

TABLA 1. Número de especies en cada tipo de vegetación, compilado de la lista de especies registradas. En los casos en que la especie ocurre en más de un tipo de vegetación, se le incluyó solamente en el más característico para la especie

\begin{tabular}{lccccc}
\hline $\begin{array}{c}\text { Tipo de } \\
\text { vegetación }\end{array}$ & $\begin{array}{c}\text { Bosque de } \\
\text { encinos }\end{array}$ & Pastizal & $\begin{array}{c}\text { Matorral } \\
\text { xerófilo }\end{array}$ & $\begin{array}{c}\text { Vegetación } \\
\text { acuática y } \\
\text { subacuática }\end{array}$ & $\begin{array}{c}\text { En los } \\
\text { alrededores } \\
\text { de áreas } \\
\text { habitadas }\end{array}$ \\
\hline $\begin{array}{l}\text { Número de especies } \\
\text { registradas } \\
\begin{array}{l}\text { Porcentaje del } \\
\text { total }\end{array}\end{array}$ & 127 & 111 & 100 & 16 & 21 \\
\hline
\end{tabular}


TABLA 2. Comparación de la diversidad florística del cerro Tetzcotzinco con la de otras áreas

\begin{tabular}{|c|c|c|c|c|c|}
\hline $\begin{array}{c}\text { Localidad y altitud } \\
\text { (msnm) }\end{array}$ & $\begin{array}{c}\text { Autor y año de } \\
\text { publicación }\end{array}$ & $\begin{array}{l}\text { Área } \\
\text { (ha) }\end{array}$ & $\begin{array}{c}\text { Número de } \\
\text { especies }\end{array}$ & $\begin{array}{l}\text { Número de } \\
\text { géneros }\end{array}$ & $\begin{array}{l}\text { Número de } \\
\text { familias }\end{array}$ \\
\hline $\begin{array}{l}\text { Pedregal de San Ángel } \\
\quad 2250-3100\end{array}$ & $\begin{array}{l}\text { J. Rzedowski } \\
\text { (1954) }\end{array}$ & 8000 & 654 & 318 & 86 \\
\hline $\begin{array}{l}\text { Sierra de Guadalupe } \\
2200-2700\end{array}$ & $\begin{array}{l}\text { M. Bopp O. } \\
(1956)\end{array}$ & 27200 & 294 & 200 & 57 \\
\hline $\begin{array}{l}\text { San Juan Tetla } \\
\qquad 3000-3600\end{array}$ & $\begin{array}{l}\text { J.C. Boyas D. } \\
(1978)\end{array}$ & 1500 & 290 & 160 & 58 \\
\hline $\begin{array}{c}\text { Cerro Tetzcotzinco } \\
2270-2600\end{array}$ & $\begin{array}{l}\text { Pulido y Koch } \\
\text { (el presente estudio) }\end{array}$ & 50 & 375 & 234 & 70 \\
\hline
\end{tabular}

todo el año. De este modo se forma un gran número de microhábitats con diferentes condiciones ambientales.

3) Perturbación. Es causada principalmente por los animales domésticos, por los intentos del hombre de aprovechar pequeñas áreas para cultivos y, aunque en menor grado, por el aprovechamiento de especies que crecen silvestres en el cerro. Paralelamente con estas actividades, se dan quemas frecuentes según se ha observado en el campo; éstas seguramente son importantes para mantener el pastizal, que desaparecería sin las quemas.

4) Conservación. Aunque puede parecer contradictorio en el inciso anterior, los habitantes de los alrededores, cuidan y tratan de conservar la vegetación ya que les es útil. Además, la mayoría de ellos tiene idea de la importancia de este sitio por la historia que se les ha heredado verbalmente de generación en generación.

Especies raras. Se encontraron varias especies que destacan por su escasez en general o por lo menos en el Valle de México. A continuación se presenta un breve análisis de cada una de ellas (el asterisco indica una especie útil):

Euphorbia graminea. Fue mencionada con anterioridad solamente para dos localidades en este Valle (Rzedowski y Rzedowski, 1979).

"Hymenocallis harrisiana. Su población en el Valle es muy reducida y está señalada como una especie en peligro de extinción; además no se había recolectado en los últimos 50 años (Rzedowski y Rzedowski, 1979).

Leonotis nepetaefolia. Es una especie introducida de África (Standley y Williams, 1973), y aunque está ampliamente distribuida en el país como maleza, no es común en los alrededores del cerro.

* Malvaviscus arboreus. En el Valle de México, se ha registrado en pocas localidades: Huehuetoca, Chapultepec y Pedregal de San Ángel (Fryxell, comunicación personal). Minuartia moehringioides. Se ha citado como especie no frecuente en el Valle de México (Rzedowski y Rzedowski, 1979).

Pavonia pulidoae. Esta especie fue registrada como nueva para la ciencia (Fryxell, 1980) y el género constituyó un registro nuevo para el Valle de México. En la localidad estudiada forma un pequeño manchón. Otro sitio en el que se ha encontrado esta especie es cerca de Ciudad Valles, San Luis Potosí (Fryxell, comunicación personal).

Polypodium arcanum. Aparentemente esta especie no se ha reportado para el Valle de México y en nuestra localidad es escasa. 
*Tecoma stans. Hay pocos individuos de esta especie en el cerro, pero no llega a considerarse rara; es común en lugares más cálidos que Tetzcotzinco. En el Valle de México ya se ha registrado, pero no como abundante.

*Thevetia peruviana. Esta especie está representada en el cerro por muy pocos individuos, todos creciendo en un área no mayor de $10 \mathrm{~m}^{2}$ pero al parecer están prosperando. Su ubicación poco accesible y el hecho de que los lugareños la desconocen, indican que no goza de cuidados humanos. Los únicos registros de esta especie en el Valle de México son tres colectas de E. Matuda [Matuda s.n., 46 y 83 (MEXU)] de Tlaltihuacan, Estado de México.

La distribución restringida en el Valle de las cuatro especies útiles, marcadas con asterisco, y su asociación con un sitio arqueológico pueden ser interpretadas como evidencia botánica de que antiguamente existió en Tetzcotzinco un jardín cultivado; es decir, que fueron traídas y cuidadas, habiendo sobrevivido en descuido por varios siglos hasta hoy. En cuanto a las causas probables para traer estas especies hasta aquí, sólo tenemos que referirnos a la grandeza cultural que alcanzó Texcoco en tiempos del rey Nezahualcóyotl (Vigil, 1979, p. 89) y a las crónicas donde los conquistadores españoles relatan su sorpresa al encontrar un gran número de especies útiles especialmente en la medicina herbolaria (Pomar, 1975, p. 64).

Sin embargo, existe también la posibilidad de que estas especies, por lo menos las de Malvaviscus, Tecoma y Thevetia, sean reliquias indicadoras de que en tiempos anteriores el clima del Valle de México era más cálido que el de hoy (Rzedowski y Rzedowski, 1979), lo que explica la presencia de Cissus sicyoides en el Valle, por cierto, una especie abundante en algunas partes del cerro.

Por otro lado, cabe la posibilidad de que estas especies, o algunas de ellas, fueran introducidas en tiempos recientes. Éste puede ser el caso con Hymenocallis harrisiana y Malvaviscus arboreus, puesto que ambas son cultivadas para ornato en los pueblos que rodean el cerro.

Sin embargo, no se puede considerar la presencia de estas especies como evidencia suficiente de que en este cerro existió un jardín cultivado en el siglo xv; solamente debe considerarse como una más de las posibles evidencias.

\section{CONCLUSIONES}

El cerro Tetzcotzinco tiene una riqueza florística excepcional, sobre todo cuando se le compara con los cerros cercanos. Esto se debe a un conjunto de factores que incluyen el clima templado y benigno de la región, la abundancia y diversidad de microhábitats, una perturbación constante pero ligera, y la tendencia a conservar el área por parte de sus habitantes.

Como parte de la riqueza florística, existen ocho especies que son escasas en el Valle de México. De éstas, cuatro son de interés especial porque son útiles como medicinales o plantas de ornato, y han sido aprovechadas desde tiempos prehispánicos. De ahí que su presencia en el cerro se interprete como reliquias de un jardín prehispánico, lo cual ha sido confirmado por análisis de códices y manuscritos (Corona, 1982). Sin embargo, existen otras hipótesis botánicas igualmente o quizá más probables. 
Lista de especies registradas, en orden alfabético por familias. Los números que siguen a cada nombre indican el tipo (los tipos) de vegetación en donde más abunda ese taxon, según lo siguiente: 1-bosque de encinos; 2-pastizal; 3-matorral xerófilo; 4-vegetación acuática y subacuática; 5-cerca de casas habitación.

AMARANTHACEAE

Alternanthera pungens H.B.K. (2)

Amarantbus bybridus L. (2)

Gomphrena pringlei Coult. \& Fisher (3)

Guillerminia densa (Willd.) Moq. (3)

Iresine calea (Ibáñez) Standl. (3)

I. grandis Standl. (2)

\section{AMARYLLIDACEAE}

Agave ferox Koch (2)

A. mapisaga Trel. (3)

Bomarea hirtella (H.B.K.) Herb. (2, 3)

Hymenocallis harrisiana Herb. (3)

Hypoxis mexicana Schultes (1)

Manfreda pringlei Rose (1)

Sprekelia formosissima (L.) Herb. (2, 3)

Zephyranthes sessilis Herb. $(1,2,3)$

\section{ANACARDIACEAE}

Schinus molle L. $(2,3)$

\section{APOCYNACEAE}

Thevetia peruviana (Pers.) K. Schum. (3)

\section{ASCLEPIADACEAE}

Asclepias linaria Cav. $(1,2)$

A. ovata Mart. \& Gal. $(1,2)$

Cynanchium kuntbii (Decaisne), Standl. (1)

Gonolobus uniflorus H.B.K. $(1,5)$

Sarcostemma elegans Decaisne (3)

BEGONIACEAE

Begonia gracilis H.B.K. (1)

\section{BETULACEAE \\ Alnus acuminata H.B.K. ssp. glabrata (Fern.) Furlow (1) \\ A. jorullensis H.B.K. spp. jorullensis (1)}

\section{BIGNONIACEAE}

Tecoma stans H.B.K. (3)

\section{BORAGINACEAE}

Lithospermum calycosum (Macbride) I.M. Johnston (3)
T. lepidosepala L.B. Smith $(1,3)$

T. recurvata $\mathrm{L} .(1,3)$

T. usneoides L. $(1,3)$

\section{CACTACEAE}

Mammillaria rhodantha Link \& Otto (3)

Opuntia byptiacantha Weber $(2,3)$

O. lasiacantha Pfeiffer $(2,3)$

O. megacantha Salm-Dyck $(1,2,3)$

CAMPANULACEAE

Diastatea micrantha (H.B.K.) McVaugh (3)

CAPRIFOLIACEAE

Symphoricarpus microphyllus

H.B.K. $(1,5)$

\section{CARYOPHYLLACEAE}

Arenaria lanuginosa (Michx.) Rohrb. in Mart. (2) Arenaria lycopodioides Willd. ex Schl. (1)

Cerastium nutans Raf. (1)

Drymaria laxiflora Benth. (1)

D. leptophylla (Cham. \& Schlecht.) Fenzi $(1,2,3)$

D. multiflora Brandegee (1)

D. tenuis Wats. (2)

Minuartia moebringioides Mattf. (1)

Silene laciniata Cav. (1)

Stellaria cuspidata Willd. $(1,2,3)$

CHENOPODIACEAE

Chenopodium album L. (3)

C. ambrosioides L. (3)

CISTACEAE

Helianthemum glomeratum Lag. (1)

COMMELINACEAE

Commelina coelestis Willd. $(1,3)$

C. dianthifolia DC. (3)

C. scabra Benth. (2)

Phaeosphaerion leiocarpum (Benth.) Hassk. (3)

Tinantia erecta (Jacq.) Schlecht. (1)

Tradescantia crassifolia Cav. $(2,3)$

Tripogandra purpurascens (Schauer) Handlos ssp. purpurasens (2) 
Ageratum corymbosum Zucc. (1)

Archibaccharis mucronata (H.B.K.), Blake $(1,5)$

Artemisia ludoviciana Nutt. ssp. mexicana (Willd.) Keck (2)

Aster subulatus Michx. $(1,2)$

Baccharis conferta H.B.K. (2)

B. multiflora H.B.K. $(1,2)$

B. pteronioides Gray (5)

B. sordescens DC. $(1,2,3)$

B. thesioides H.B.K. $(2,5)$

Bidens aurea (Ait.) Sherff $(1,5)$

B. odorata Cav. var. calcicola (Greenm.) Ball $(1,3)$

B. odorata Cav. var. odorata (3)

Brickellia pendula Gray (5)

B. veronicifolia (DC.) Gray (2)

Conyza bonariensis (L.) Cronquist (2)

C. canadensis (L.) Cronquist (3)

C. coronopifolia H.B.K. (1)

C. filaginoides (A. DC.) Hieron. (1)

C. sophiifolia H.B.K. (3)

Cosmos bipinnatus Cav. (2)

Dablia coccinea Cav. $(1,3)$

D. merckii Lehm. (2)

D. rudis Sorensen $(1,3)$

Dyssodia papposa (Vent.) A.S. Hitchc. (3)

Erigeron longipes DC. (2)

Eupatorium aschenbornianum Schauer (1)

E. deltoideum Jacq. (1)

E. isolepis Robins. (1)

E. petiolare Moc. $(2,3)$

E. pycnocephalum Less. (5)

Florestina pedata Cass. (3)

Galinsoga parviflora Cav. $(1,3)$

G. quadriradiata R. \& P. $(1,3)$

Gnaphalium americanum Mill. (2)

G. arizonicum Gray (2)

G. oxyphyllum DC. (2)

G. rosaceum I.M. Johnst. (3)

G. semiamplexicaule DC. (2)

G. viscosum H.B.K. (3)

Gymnosperma glutinosum (Spreng.) Less. (2)

Haplopappus venetus (H.B.K.) Blake (2)

Heterosperma pinnatum Cav. $(2,3)$

Montanoa tomentosa Cerv. $(2,3)$

Pinaropappus roseus Less. (2)

Piqueria trinervia Cav. (2)

Sanvitalia procumbens Lam. (2)

Schkubria pinnata (Lam.) O. Ktze. var. virgata (Llave) Heiser (2)

Senecio praecox (Cav.) DC. var. praecox (2)

Senecio roldana DC. (1)

S. salignus DC. (2)

Simsia amplexicaulis (Cav.) Pers. (3)

Sonchus oleraceus L. $(2,3)$

Stevia jorullensis H.B.K. (1)

S. micrantha Lag. (3)
S. ovata Willd. var. ovata $(1,3)$

S. porphyrea McVaugh (2)

S. salicifolia Cav. (2)

S. serrata Cav. (2)

S. tephrophylla Blake (1)

S. tomentosa H.B.K. (1)

Tagetes erecta L. (2)

T. lunulata Ort. $(2,3)$

T. micrantha Cav. (2)

Taraxacum officinale Weber $(2,3)$

Tithoniu tubaeformis (Jacq.) Cass. $(2,3)$

Verbesina virgata Cav. (1)

Viguiera excelsa (Willd.) Benth. \& Hook. (2)

Zaluzania augusta Sch. Bip. (2)

Zinnia peruviana (L.) L. (3)

\section{CONVOLVULACEAE}

Cuscuta applanata Engelm. (1)

Dichondra argentea H.B.K. (2)

Ipomoea decasperma Lam. (3)

I. dumetorum Willd. (3)

I. purpurea (L.) Roth var. diversifolia (Lindl.)

O'Donnell $(2,3)$

I. stans Cav. $(1,2)$

I. trifida (H.B.K.) G. Don (2)

\section{CRASSULACEAE}

Sedum greggii Hemsl. (1)

S. longipes Rose (1)

S. moranense H.B.K. (1)

S. praealtum (A. DC.) Clausen ssp. parvifolium Clausen (3)

S. quevae Hamet (3)

\section{CRUCIFERAE}

Brassica campestris L. (2)

Descurainia impatiens (Cham. \& Schl.) O.E. Schultz (3)

Eruca sativa Mill. (2)

Halimolobos hispidula (DC.) Schultz (1)

Lepidium virginicum $\mathrm{L}$. (2)

Pennellia micrantha (Gray) Nieuwl. (2)

Rorippa nasturtium-aquaticum (L.) Schinz \& Thell. $(4,5)$

CUCURBITACEAE

Cyclanthera ribiflora (Schlecht.) Cogn. $(1,2)$

Sicyos parviflora Willd. (1)

CUPRESSACEAE

Juniperus deppeana Steud (1)

CYPERACEAE

Bulbostylis juncoides (Vahl) Kükenth. (2)

Cyperus flavus (Vahl) Nees (1) 
C. manimae H.B.K. (2)

C. niger Ruiz et Pavón (4)

C. seslerioides H.B.K. $(2,3)$

C. spectabilis Link $(2,3)$

C. tenuis R. \& P. $(2,3)$

\section{DIOSCOREACEAE}

Dioscorea galeottiana Kunth $(2,3)$

EUPHORBIACEAE

Acalypha indica L. $(2,3)$

Euphorbia dentata Michx. (2)

E. graminea Jacq. (1)

E. prostrata Ait. $(2,3)$

Stillingia zelayensis (H.B.K.) Muell. Arg. (2)

\section{FAGACEAE}

Quercus deserticola Trel. (1)

Q. frutex Trel. (1)

Q. rugosa Neé (1)

\section{GERANIACEAE}

Erodium cicutarium (L.) L'Her. (2)

Geranium aristisepalum Moore (1)

G. seemannii Peyr. (2)

\section{GRAMINEAE}

Aegopogon cenchroides H. \& B. ex Willd. (2) Agrostis semiverticillata (Forsk.) Christ. (4)

Aristida adscensionis L. (2)

A. divaricata Humb. \& Bonpl. (2)

Avena fatua L. (3)

Botbriochloa saccharoides (Swartz) Rydb. (2)

Boutelona curtipendula (Michx.) Torr. (2)

B. gracilis (H.B.K.) Lag. (3)

B. simplex Lag. (3)

Brachiaria meziana A.S. Hitchc. (2)

Briza subaristata Lam. $(1,5)$

Bromus anomalus Rupr. ex Fourn. $(2,3)$

B. carinatus Hook. \& Arn. $(2,3,5)$

Chloris submutica H.B.K. (2)

Echinochloa crus-galli (L.) Beauv. (4)

E. oplismenoides (Fourn.) A.S. Hitchc. (4)

Eragostis intermedia Hitch. $(1,2)$

E. mexicana (Hornem.) Link ssp. mexicana $(1,3)$

E. pectinacea (Michx.) Nees. (2)

Hilaria cenchroides H.B.K. (2)

Leptocbloa dubia (H.B.K.) Nees (2)

Lycurus phleoides H.B.K. (2)

Microchloa kuntbii Desv. (2)

Mublenbergia distans Swallen (1)

M. implicata (H.B.K.) Kunth (2)

M. microsperma (DC.) Kunth (2)

M. pusilla Steudel (2)

M. ridiga (H.B.K.) Kunth (1)
M. robusta (Fourn.) A.S.Hitchc. $(1,2)$

Nassella linearifolia (Fourn.), Pohl (1)

Panicum bulbosum H.B.K. (2)

Paspalum convexum Humb. \& Bonpl. (2)

Peyritschia koelerioides (Peyr.), Fourn. (2)

Piptochaetium fimbriatum (H.B. K.) A.S. Hitchc. (2)

Polypogon interruptus H.B.K. (4)

Schizachyrium birtiflorum Nees (2)

Setaria gracilis Kunth. (5)

S. grisebachii Fournier (3)

Sporobolus poiretii (R. \& S.) A.S. Hitchc. (2)

Stipa constricta A.S. Hitchc. (3)

S. ichu (R. \& P.) Kunth (1)

S. mucronata H.B.K. $(1,3)$

S. virescens H.B.K. (1)

Vulpia myuros (L.) Gmelin var. birsuta Hack. (2)

V. myuros (L.) Gmelin var. myuros $(1,2)$

HYDROPHYLLACEAE

Nama dichotomum (R. \& P.) Choisy var. chasmogamum Brand. (1)

IRIDACEAE

Nemastylis tenuis (Baker) Benth. (1)

Sisyrinchium bracteatum Greenm. $(1,2)$

S. schaffneri Wats. $(1,2)$

Tigridia vanhoutei Roezl $(2,3)$

JUNCACEAE

Juncus dichotomus Ell. (4)

J. imbricatus Leharpe (4)

LABIATAE

Leonotis nepetaefolia A. Br. (2)

Marrubium vulgare L. $(2,5)$

Rosmarinus officinalis L. (5)

Salva amarissima Ort. (2)

S. laevis Benth. (1)

S. mexicana L. $(2,5)$

S. microphylla H.B.K. (1)

S. polystachya Ort. $(1,2)$

S. tiliifolia Vahl (3)

Stachys coccinea Jacq. (1)

S. nepetifolia Desvaux (2)

\section{LEGUMINOSAE}

Astragalus strigulosus H.B.K. (3)

Brongniartia intermedia Moric. (3)

Calliandra grandiflora (L'Her.) Benth. (3)

Cassia tomentosa L.f. $(3,5)$

Cologania grandiflora Rose (2)

Crotalaria pumila Ort. (2)

Dalea foliolosa (Ait.) Barneby var. foliolosa (2)

D. minutifolia (Rydb.) Harms (1)

D. reclinata (Cav.) Willd. (1) 
D. zimapanica Schauer $(2,3)$

Desmodium grahamii Gray (1)

D. neomexicanum Gray (2)

D. uncinatum (Jacq.) DC. (1)

Erythrina leptorbiza DC. (3)

Eysenhardtia polystachya (Ort.), Sarg. (3)

Macroptilium heterophyllum (Willd.)

Maré. \& Baudet $(1,2)$

Medicago lupulina L. (3)

M. polymorpha L. (2)

Mimosa biuncifera Benth. (2)

Phaseolus coccineus L. $(1,2,3)$

P. formosus H.B.K. (3)

P. pluriflorus Maré, Masch. \& Stain (1)

Trifolium amabile H.B.K. (2)

Zornia thymifolia H.B.K. (2)

\section{LENTIBULARIACEAE}

Pinguicula moranensis H.B.K. (1)

\section{LILIACEAE}

Allium rhizomatum Woot. \& Standl. (3)

Calochortus barbatus (H.B.K.), Painter (1)

Echeandia mexicana Cruden (2, 3)

E. nana (Baker) Cruden (1)

Milla biflora Cav. (2)

Schoenocaulon coulteri Baker (1)

LOASACEAE

Mentzelia bispida Willd. $(2,3)$

LOGANIACEAE

Buddleia cordata H.B.K. ssp. cordata (2)

B. sessiliflora H.B.K. (2)

\section{LORANTHACEAE}

Phoradendron brachystachyum (DC.), Nutt. (3)

LYTHRACEAE

Cuphea aequipetala Cav. $(2,3)$

C. wrightii Gray $(2,3)$

MALPIGHIACEAE

Gaudichaudia cynanchoides H.B.K. (3)

MALVACEAE

Abutilon ellipticum Schlecht. (2)

Anoda cristata (L.) Schlecht. $(2,3)$

Kearnemalvastrum subtriflorum (Lag.) Bates (2)

Malvaviscus arboreus Cav. (2)

Pavonia pulidoae P.A. Fryxell (3)

Cannabis sativa L. (2)
NYCTAGINACEAE

Mirabilis jalapa L. (3)

M. longiflora L. (1)

OLEACEAE

Fraxinus uhdei (Wenz.) Ling. $(2,5)$

ONAGRACEAE

Epilobium mexicanum DC. (4)

Gaura coccinea Nutt. $(2,3)$

Lopezia racemosa (Cav. $(1,3)$

Oenothera pubescens Willd. ex Spreng. (2)

O. rosea Ait. (2)

O. tetraptera Cav. (2)

ORCHIDACEAE

Malaxis ehrenbergii (Reichb.) Kuntze (1)

Spiranthes sp. (2)

S. polyantha Reichb. $(1,2,3)$

S. schaffneri Reichb. (1)

\section{OXALIDACEAE}

Oxalis albicans H.B.K. (2)

O. alpina (Rose) Kunth (2)

O. corniculata L. (3)

O. divergens Benth. ex Lindley (2)

$O$. herdandesii DC. (3)

O. latifolia H.B.K. (2)

O. Iunulata Zucc. (2)

O. tetraphylla Cav. var. tetraphylla (3)

PAPAVERACEAE

Argemone ochroleuca Sweet ssp. ochroleuca (2)

PHYTOLACCACEAE

Phytolacca icosandra L. $(2,3)$

PIPERACEAE

Peperomia campylotropa Hill (1)

PLANTAGINACEAE

Plantago australis Lam. ssp. birtella (H.B.K.) Rahn (2)

P. nivea H.B.K. $(2,3)$

PLUMBAGINACEAE

Plumbago pulchella Boiss. $(2,3)$

POLEMONIACEAE

Loeselia coerulea (Cav.) Don (2)

L. glandulosa (Cav.) Don $(1,2)$

L. mexicana Brand $(2,3)$

POLYGONACEAE

Polygonum lapathifolium L. (4) 
Rumex crispus L. (5)

R. pulcher L. (3)

\section{POLYPODIACEAE}

Adiantum andicola Liebm. (2)

A. capillus-veneris L. (2)

A. poiretii Wikstr. (2)

Asplenium monanthes L. (1)

Cheilanthes hirsuta Link (1)

C. myriophylla Desv. $(2,3)$

Cystopteris fragilis Bernh. (1)

Notholaena aurea (Poir.) Desv. $(2,3)$

N. sinuata (Lag.) Kaulf. var. integerrima Hook. $(2,3)$

Pellaea ovata (Desv.) Weatherby $(2,3)$

P. ternifolia (Cav.) Link (1)

Pleopeltis polylepis (Roem. ex Kunze)

Moore var. polylepis (1)

Polypodium arcanum Maxon (1)

P. subpetiolatum Hook. (1)

P. thyssanolepis A. Br. (1)

Woodsia mollis (Kaulf.) J. Sm. (1)

PORTULACACEAE

Portulaca mexicana P. Wilson $(2,3)$

Talinum napiforme DC. (1)

\section{PRIMULACEAE}

Anagallis arvensis L. (2)

Centunculus minumus L. (4)

\section{RANUNCULACEAE}

Ranunculus dichotomus Moc. \& Sessé (4)

R. machranthus Scheele (4)

Thalictrum strigillosum Hemsl. $(1,3)$

\section{RESEDACEAE}

Reseda luteola L. (2, 3)

ROSACEAE

Alchemilla pringlei Fedde (2)

Amelanchier denticulata (H.B.K.) Koch $(1,2)$

Crataegus pubescens (H.B.K.) Steud. (5)

Prunus micropbylla (H.B.K.) Hemsl. $(3,5)$

P. persica (L.) Sieb. \& Zucc. $(3,5)$

P. serotina Ehrh. ssp. capulli (Cav.) McVaugh (2)

Pyrus malus L. (5)

\section{RUBIACEAE}

Bouvardia longiflora Gray $(2,3)$

B. ternifolia (Cav.) Schlecht. $(1,2)$

Crusea diversifolia (H.B.K.) W. R. Anderson (1, 2)

C. longiflora (Willd. ex. R. \& S.) W.R. Anderson (1)

Galium aschenbornii Schauer (1)
G. uncinulatum DC. (1)

Richardia tricocca Schumann ssp. tetracocca (Mart. \& Gal.) Lewis \& Oliver $(2,4)$

RUTACEAE

Casimiroa sapota Oerst. (3)

Ruta chalapensis L. (5)

\section{SCROPHULARIACEAE}

Bacopa procumbens (Mill.) Greenm. (2)

Lamourouxia dasyantha (Cham. et Schlecht.) W.R. Ernst (1)

L. multifida H.B.K. (1)

Mimulus glabratus H.B.K. (4)

Penstemon roseus (Sweet) G. Don $(1,2)$

SELAGINELLACEAE

Selaginella pallescens (Presl.) Spring (1)

S. peruviana (Milde) Hieron. $(1,2)$

\section{SOLANACEAE}

Datura stramonium L. (2)

Jaltomata procumbens (Cav.) J. Gentry (1)

Nicotiana glauca Graham (3)

Physalis chenopodiifolia Lam. (1)

Solanum bulbocastanum Dun. (1)

S. cervantesii Lag. (2)

S. douglasii Dunal (1)

S. laurifolium Mill. (3)

\section{UMBELLIFERAE}

Apium leptophyllum F. Muell. (3)

Eryngium comosum Delar f. (2)

Lilaeopsis schaffneriana (Schlecht.) C. \& R. (4)

Prionosciadium thapsoides (DC.) Mathias (3)

URTICACEAE

Parietaria pensylvanica Muhl. (1)

VALERIANACEAE

Valeriana densiflora Benth. (1)

V. sorbifolia H.B.K. var. mexicana (DC.) G.F. Mey. (1)

\section{VERBENACEAE}

Priva mexicana (L.) Pers. (3)

Verbena carolina L. (2)

$V$. ciliata Benth. (2)

$V$. gracilis Desf. (3)

V. menthifolia Benth. $(2,3)$

VITACEAE

Cissus sicyoides L. (3) 
AGRADECIMIENTOS. Los autores desean expresar su sincero agradecimiento a las numerosas personas que ayudaron en la realización de esta investigación. Entre ellas cabe mencionar al Dr. J. Rzedowski y al M. en C. M. Sousa, por permitir el acceso a los herbarios a su cargo; a los doctores H. Bravo-Hollis, P.A. Fryxell, S. González E., C. Rodríguez J., T. Wendt y R. Galván V.: a los biólogos F.J. Espinosa G. y J.L. Villaseñor R., y al señor J. García P., por la identificación o revisión de ejemplares; al etnólogo E. Corona S., por sus consejos y su interés en el proyecto. Además, agradecemos al Consejo Nacional de Ciencia y Tecnología y al Colegio de Postgraduados de Chapingo por su apoyo económico. A pesar de lo anterior, los autores aceptan toda la responsabilidad por cualquier error en el presente trabajo.

\section{LITERATURA CITADA}

Alva IXtlixóchitl, F., de 1955, Obras Históricas.. Reimpresión fotográfica, Universidad Nacional Autónoma de México, México, vol. 2, Historia Chichimeca.

Bopp O., M. 1956. Contribución al estudio de la flora fanerogámica de los cerros situados al norte de la ciudad de México. Tesis, Facultad de Ciencias, Universidad Nacional Autónoma de México, México, 65 pp.

BOYAS, J.C. 1978. Contribución al conocimiento de la flora fanerogámica del Campo Experimental Forestal "San Juan Tetla", estado de Puebla. Tesis, Escuela de Ciencias Biológicas, Universidad Autónoma del Estado de Morelos, Cuernavaca, 243 pp.

Cetenal. 1979. Carta Geológica de Chalco, México y Distrito Federal. 1:50 000. Coordinación General de Estudios del Territorio Nacional, México.

- 1979a. Carta Geológica de Texcoco, México y Tlaxcala. 1:50 000. Coordinación General de Estudios del Territorio Nacional, México.

Corona, J.E. 1978. Las terrazas de Nezahualcóyotl. INIreB Informa 22:1-2.

. 1982. El recurso lacustre en la formación Acolhua. Bol. Esc. Cienc. Antropol. Univ. Yucatán 55:3-12.

Fryxell, P.A. 1980. Three new species of Malvaceae from Mexico. Phytologia 46 (6): 391-398.

GARCíA, E. 1978. Los climas del Valle de México según el sistema de clasificación climática de Köeppen modificado por la autora, 2a. reimpresión. Colegio de Postgraduados, Chapingo, México, 39 pp.

Maldonado Koerdell, M. 1941. Los jardines botánicos de los antiguos mexicanos. Rev. Soc. Mex. Hist. Nat. 2:79-84.

Mendizábal, M.O., de. 1925. El jardín de Nezahualcóyotl. Ethnos. (Méx.) Ser. III 1(3-4):86-95.

Ortiz S., C.A. y H. Cuanalo DE la Cerda. 1977. Levantamiento fisiográfico del área de influencia de Chapingo. Colegio de Postgraduados, Chapingo, México, 83 pp.

POMAR, J.B. 1975. Relación de Texcoco. Edición facsimilar de la de 1891, con advertencia, preliminar y notas de Joaquín García Icazbalceta. Biblioteca Enciclopédica del Estado de México 49, México, 69 pp.

Rzedowski, J. 1954. Vegetación del Pedregal de San Ángel (México). An. Esc. Nal. Cienc. Biol. Méx. 8:59-129.

y G.C. DE RzEDOWSKI. 1979. Flora fanerogámica del Valle de México. Compañía Editorial Continental, México, vol. 1.

Standley, P.C. y L.O. Williams. 1973. Labiatae. En: Flora of Guatemala. Fieldiana Bot. 24(9):237-317.

VigIL, J.M. 1979. Nezahualcóyotl. Edición facsimilar de la de 1972, preparada por Ernesto Lemoine. Biblioteca Enciclopédica del Estado de México 71, México, 133 pp. 